REGARDS

SUR LECONOMIE ALLEMANDE

BULLETIN ECONOMIQUE DU CIRAC
Regards sur l'économie allemande

Bulletin économique du CIRAC

$88 \mid 2008$

Varia

\title{
Les Sparkassen, facteur de stabilité pour l'économie allemande
}

\section{Karl-Peter Schackmann-Fallis}

Traducteur : Isabelle Bourgeois

\section{OpenEdition \\ Journals}

Édition électronique

URL : http://journals.openedition.org/rea/1103

DOI : $10.4000 /$ rea. 1103

ISBN : 978-2-8218-0872-0

ISSN : 1965-0787

Éditeur

CIRAC

Édition imprimée

Date de publication : 1 octobre 2008

Pagination : 5-14

ISSN : 1156-8992

Référence électronique

Karl-Peter Schackmann-Fallis, «Les Sparkassen, facteur de stabilité pour l'économie allemande »,

Regards sur l'économie allemande [En ligne], 88 | octobre 2008, mis en ligne le 01 octobre 2010, consulté le 30 avril 2019. URL : http://journals.openedition.org/rea/1103 ; DOI : 10.4000/rea.1103 


\title{
Les Sparkassen, facteur de stabilité pour l'économie allemande
}

\author{
Karl-Peter Schackmann-Fallis
}

\begin{abstract}
L'Allemagne aussi ressent l'impact de la crise financière internationale. A l'été 2007, la banque publique IKB, filiale de la Kreditanstalt für Wiederaufbau et dédiée au financement des PME, avait fait l'objet d'une opération de sauvetage de la part des pouvoirs publics, de même que la banque régionale publique du Land de Saxe, la Sachsen LB, avait dû être absorbée in extremis par son homologue du Bade-Wurtemberg, la LBBW. En ce début d'automne 2008, la banque immobilière Hypo Real Estate a été de justesse sauvée de la faillite. Néanmoins, la liste des dommages collatéraux de l'éclatement de la bulle spéculative semble pour l'instant se limiter à ces trois cas, aux dires du gouvernement fédéral. Si le secteur bancaire allemand est exposé comme tous les autres aux effets indirects de la crise des subprimes, et si aucun de ses trois piliers (banques privées, coopératives et caisses d'épargne) n'est totalement à l'abri, il n'en jouit pas moins d'une constitution robuste. Et celle-ci, en prémunissant largement les entreprises, et tout particulièrement celles du Mittelstand sur lequel repose en grande partie la compétitivité de l'économie allemande (voir REA 83/07), du risque d'un resserrement du crédit, comme l'expliquent aussi bien la Bundesbank que la fédération de l'industrie $B D I$, réduit l'impact des turbulences de la finance mondiale sur la croissance allemande.
\end{abstract}

Ces turbulences révèlent a contrario les éléments structurels à l'origine de cette stabilité. Dans la déclaration gouvernementale sur l'état des marchés financiers faite devant le Bundestag le 25-09-2008, le ministre fédéral des Finances, Peer Steinbrück, les résumait ainsi : " cette crise... fait apparaître que le système de la banque universelle avec ses trois piliers..., un système conforme à notre modèle d'économie sociale de marché, est nettement plus solide que le système angloaméricain avec son principe de la banque mono-métier et sa fixation sur une rentabilité à court terme ". Dans ce système, le rôle principal de 'stabilisateur' de l'économie revient à deux piliers : à celui des banques coopératives et, plus encore au réseau des caisses d'épargne, expressément salué par Peer Steinbrück pour sa culture orientée sur le long terme et son ancrage dans l'économie réelle.

Bien sûr, ce constat a pour fonction de rassurer l'opinion. Mais il repose sur la réalité d'indicateurs macro-économiques et d'analyses structurelles incontestables. Il a également pour propos, à l'heure où s'avive le conflit qui oppose l'Allemagne et la Commission européenne sur le dossier (aides indues d'Etat) de la banque publique de Rhénanie du Nord-Westphalie, la West LB, de plaider vis-àvis de 'Bruxelles' la cause de ces banques qui sont un élément constitutif du réseau des caisses d'épargne et de forcer en interne une rapide réforme de leurs structures et missions.

Nous avons demandé à M. Schackmann-Fallis, membre du bureau exécutif de la Fédération allemande des Caisses d'épargne (Deutscher Sparkassen- und Giroverband), qui en préside la direction Economie et politique et est en charge également des questions européennes et des affaires internationales, de nous dresser un état des lieux du marché bancaire allemand et de nous exposer la place particulière qu'y occupe le réseau des caisses d'épargne. (IB) 
Le rachat de la Dresdner Bank par la Commerzbank, puis l'entrée de Deutsche Bank au capital de la Postbank à la fin de l'été ont attiré l'attention internationale sur le site bancaire allemand. Ces deux 'méga-fusions' intervenues coup sur coup, et dont l'une préfigure la privatisation de la banque postale allemande, révèlent en effet l'accélération du mouvement de concentration dans le segment des banques privées, l'un des trois piliers du secteur bancaire allemand. Or les évolutions observées dans ce secteur ne sont elles aussi que la manifestation des profondes mutations qui affectent depuis de nombreuses années l'ensemble des activités en Allemagne.

\section{Un marché bancaire diversifié malgré la prédominance du modèle de banque universelle}

Le système allemand présente trois principales caractéristiques. La première est la prédominance du modèle de la banque universelle, c'est-à-dire multiservices et multi-clients, dont l'offre est par conséquent peu spécialisée. La seconde est une grande diversité d'acteurs tant du point de vue qualitatif que quantitatif, autrement dit : la coexistence entre grands groupes déployant leurs activités sur l'ensemble du territoire et instituts de taille petite à moyenne oeuvrant à l'échelon local ou régional. La troisième est l'articulation entre différents statuts juridiques et modèles économiques, communément résumée par l'image d'un système à trois piliers : banques privées, banques coopératives et caisses d'épargne publiques.

Structure du système bancaire allemand à la fin 2007

\begin{tabular}{|lrrr|}
\hline Nombre de... & $\ldots$ banques & $\ldots$ filiales & $\ldots$ salariés \\
Total & $\mathbf{2 0 1 2}$ & $\mathbf{3 7 9 7 6}$ & $\mathbf{6 6 2} 650$ \\
Banques de crédit privées & 278 & 11286 & $190250^{\text {b) }}$ \\
- Grandes banques & 5 & 8568 & - \\
- Banques régionales & 174 & 2628 & - \\
- Banques étrangères & 99 & 90 & - \\
Landesbanken & 12 & 485 & 39850 \\
Caisses d'épargnes & 446 & 13624 & 253700 \\
Caisses centrales coopératives & 2 & 11 & 4900 \\
Coopératives de crédits & 1234 & 12477 & 160750 \\
Banques hypothécaires publiques et privées & 22 & 64 & $\left.-{ }^{c}\right)$ \\
Banques aux missions spécifiques (dont la KfW) & 25 & 1801 & 17000 \\
\hline
\end{tabular}

Source des données : Deutsche Bundesbank, Monatsbericht, septembre 2008. a) équivalent temps plein, sans les effectifs de la Bundesbank; b) total du secteur privé ; c) les salariés des banques hypothécaires privées sont comptabilisés dans le total des effectifs du secteur privé, ceux des banques hypothécaires privées, dans le total des effectifs des banques aux mission spécifiques.

Trois piliers : le secteur privé , dominé par 5 'grandes' banques...

... un réseau de banques coopératives...
Dans le groupe des banques de crédit privées se distinguent, du moins jusqu'à présent, cinq instituts généralement désignées sous le terme de 'grandes banques'. II s'agit de la Deutsche Bank, de la Postbank (qui appartient toujours à la Deutsche Post AG, en cours de privatisation), de la Hypovereinsbank (désormais aux mains du groupe italien UniCredit), de la Commerzbank et, enfin, de la Dresdner Bank qui appartenait jusqu'à récemment à l'assureur Allianz. Toujours dans le secteur privé, on recense en outre un grand nombre d'instituts régionaux et de banques familiales, à quoi il faut ajouter de nombreuses filiales et succursales d'établissements étrangers.

Le second pilier est celui des banques coopératives ou mutualistes (Volksbanken et Raiffeisenbanken). II se compose d'instituts indépendants, reliés en un réseau d'étroite coopération par l'intermédiaire de leurs fédérations et de leurs structures communes - principalement les deux grandes centrales coopératives que sont la DZ-Bank (Deutsche Zentral-Genossenschaftsbank) et la WGZ-Bank (Westdeutsche Genossenschafts-Zentralbank). 
Le troisième pilier est celui des caisses d'épargnes (Sparkassen), organisées elles aussi en réseau : la Sparkassen-Finanzgruppe, groupe leader sur le marché bancaire allemand. II se compose d'une part des 446 caisses d'épargne communales spécialisées dans la banque de détail à l'échelon local et, d'autre part, des 7 banques publiques régionales (Landesbanken), des banques universelles dont le tour de table varie certes d'un établissement à l'autre, mais où figurent toujours les caisses d'épargne, les Länder, ainsi que des tiers (pour la plupart des actionnaires institutionnels publics - mais non étatiques -, et parfois des investisseurs privés). Ce réseau, public, comprend également la Dekabank, prestataire de services financiers et gestionnaire d'actifs centralisé, les caisses régionales d'épargne immobilière (Landesbausparkassen), les assureurs publics, ainsi que diverses autres entreprises. Cette structure en réseau permet au groupe des caisses d'épargne de présenter l'ensemble de la gamme des métiers et services bancaires.

Si on considère l'ensemble de l'activité bancaire, la Sparkassen-Finanzgruppe avait à la fin 2007 une part de marché de 35,4\% (Deutsche Bundesbank, 2008), en Allemagne, dont plus de la moitié est imputable aux Landesbanken et une petite moitié, aux caisses d'épargne. Les banques coopératives réunissent pour leur part $11,7 \%$ de parts de marché, les banques de détail privées $29,6 \%$, dont $18,4 \%$ sont à mettre à l'actif des grandes banques. Le petit quart restant se répartit entre les caisses régionales d'épargne immobilière, les banques de crédit hypothécaire et les banques publiques aux missions spécifiques (comme la Kreditanstalt für Wiederaufbau, KfW).

Si on prend pour référence les seules activités de hors-bilan, alors les parts de marché se répartissent quelque peu différemment: les grandes banques privées ont traditionnellement une présence forte dans le segment des opérations sur valeurs mobilières, ce que révèle notamment la marge globale d'intermédiation. Selon les données publiées par la Bundesbank sur cet indicateur, les grandes banques ont réalisé à elles seules en 2006 non moins de 34,8 \% du total de la marge réalisée dans ce segment. Mais de ce fait, elles sont aussi plus directement touchées par les restrictions prévisibles des activités d'intermédiation dans la période de turbulences consécutive à la crise des subprimes. A moyen terme en effet, les activités comme la titrisation ou le financement des fusions et acquisitions ne devraient connaître qu'un volume bien en deçà des seuils vertigineux enregistrés dans les années d'avant 2006, c'est-à-dire avant la crise.

En revanche, les caisses d'épargne et les banques coopératives sont, elles, très fortement représentées dans le segment de la banque de détail. Ainsi, la Sparkassen-Finanzgruppe atteignait en 2007 une part de marché de 40,4\% en ce qui concerne les encours de dépôt de la clientèle des particuliers. Avec 42 millions de comptes courants, les caisses d'épargne sont incontestablement en tête pour ce qui est des transactions financières.

Le même constat vaut également pour le financement des entreprises. A la fin 2007 , les caisses d'épargne et les Landesbanken géraient $43,3 \%$ des crédits accordés outre-Rhin aux entreprises et travailleurs indépendants. Ces instituts jouent un rôle encore plus important dans le financement du Mittelstand. L'artisanat allemand est ainsi financé à plus des deux tiers $(67,4 \%)$ par les caisses d'épargne et les Landesbanken.

Cela étant, le marché bancaire allemand est non seulement très diversifié, mais aussi largement ouvert. Tous les instituts, quel que soit leur statut, ont en effet la possibilité d'offrir l'ensemble de la gamme des prestations et de s'adresser à toutes les clientèles-cibles. Le marché n'est donc pas segmenté et ne connaît pas non plus d'exclusivité-produit comme c'est le cas par exemple en France avec le Livret $A$. Le marché bancaire allemand, libéralisé plus tôt que d'autres en Europe, est soumis à une très forte concurrence.
... et un réseau public de caisses d'épargne, leader du marché

Sparkassen-Finanzgruppe : plus du tiers du marché

Prédominance des banques privées dans le hors bilan...

... des caisses d'épargne et des banques coopératives dans le retail banking

Sparkassen et Landesbanken financent surtout le Mittelstand 
Coûts-clients avantageux mais faibles marges

Tendance à la concentration

$80 \%$ de Sparkassen e de banques coopératives

Une densité de guichets inférieure à la moyenne de la zone euro

La finance est une des principales branches de l'économie allemande

\section{Un marché où la concurrence profite au client}

Foncièrement ouvert, le marché bancaire allemand a attiré un grand nombre d'opérateurs étrangers qui s'y sont implantés avec succès comme le néerlandais ING, présent en Allemagne principalement sous l'enseigne ING-Diba. De même, le français Crédit Mutuel vient d'y reprendre les activités de détail de Citigroup. Tout concept innovant y trouve sa place : aujourd'hui (fin 2007), dans le segment de la clientèle des particuliers, les banques étrangères ont conquis une part de marché de $16,7 \%$ pour les dépôts à vue, de $20,5 \%$ pour les dépôts à court terme et de $26,7 \%$ pour les crédits à tempérament.

Du fait de la très vive concurrence, le coût des services bancaires est très avantageux pour la clientèle, ce qui tasse évidemment les marges ou la rentabilité des banques comme des caisses d'épargne. C'est ce que révèlent par exemple les statistiques de la BCE relatives aux taux d'intérêt des instituts financiers (BCE, 2008). De son côté, le cabinet d'audit et de conseil Oliver Wyman a réalisé une série d'études étayant le lien entre les faibles marges des instituts et les faibles coûts des prestations pour le client pratiqués en Allemagne (Oliver Wyman, 2008 et Mercer Oliver Wyman, 2007).

Or le marché bancaire allemand présente une autre caractéristique encore : il subit une profonde mutation. Depuis le début des années 1990, le nombre des instituts s'est réduit de moitié, passant de plus de 4000 à un peu plus de 2000 désormais. L'essentiel du mouvement de fusions-acquisitions qui explique cette réduction s'est fait dans le secteur des caisses d'épargne et celui des banques coopératives. La tendance se poursuit: depuis le début du millénaire, le nombre des banques s'est réduit d'un tiers. Non seulement, les mutations sont rapides, mais le processus de concentration en cours en Allemagne est l'un des plus dynamiques d'Europe.

Pourtant, avec 2050 instituts recensés par la BCE en 2006 (BCE, 2007), c'est en Allemagne que les banques sont les plus nombreuses en comparaison européenne. La même année, la France par exemple n'en comptait que 829. Mais ces chiffres ne sont guère significatifs si on ne tient pas compte également du rôle particulier imparti en Allemagne aux deux réseaux que sont les caisses d'épargne et les banques coopératives : à eux seuls, ils réunissent en effet près de $80 \%$ de tous les instituts bancaires recensés outre-Rhin. Certes, du point de vue juridique (et donc aussi statistique), ces instituts sont des entreprises indépendantes, mais leur rayon d'action ne dépasse pas l'échelon local ou régional. De même, ils ne sont présents sur le marché que sous une seule marque et sont liés au sein de leurs réseaux respectifs par une étroite coopération dans les activités de back-office. A y regarder de près, le marché bancaire allemand est donc loin d'être aussi fragmenté qu'on le dit communément ou comme le laisse à penser une lecture superficielle des données statistiques.

La même conclusion s'impose quand on replace le système bancaire allemand dans son contexte économique ou démographique. Alors que dans la zone euro, on dénombre près de 20 banques par million d'habitants, cette densité est supérieure en Allemagne, où on en compte presque 25. II n'y a là toutefois rien d'exceptionnel, ni en comparaison européenne, ni en comparaison internationale puisque les Etats-Unis, par exemple, présentent une densité de 28 instituts par million d'habitants.

$\mathrm{Si}$ on considère non pas le nombre de banques, mais de succursales, alors l'Allemagne se place en dessous de la moyenne de la zone euro (un peu plus de 57 guichets pour 100000 habitants), avec une densité de 49 succursales pour 100000 habitants. A titre de comparaison, la France présente une densité d'un peu plus de 63 pour 100000 (données BCE, état : fin 2006). Rien ne permet donc d'affirmer que l'Allemagne serait ,surbancarisée'.

Les activités bancaires sont une des principales branches de l'économie allemande. Avec quelque 670000 emplois, elles occupent $2 \%$ des actifs et gé- 
nèrent 3,4 \% du PIB (Initiative Finanzstandort Deutschland, 2008). En termes de création de richesse, avec 100 milliards $€$ en 2006, le secteur de la finance, qui englobe banque et assurance, surclasse même en importance la construction mécanique (73,6 milliards $€$ ) ou l'industrie automobile (71,0 milliards $€$ ).

Dans une étude qui avait largement retenu l'attention lors de sa présentation voici trois ans, la $\mathrm{KfW}$ avait démontré les remarquables progrès réalisés par la finance allemande en matière de productivité depuis le début des années 1990 (KfW-Research, 2005). Etablie sur la base d'indicateurs macro-économiques, cette étude comparative internationale (USA, Japon, Allemagne, France, Italie, Espagne, Pays-Bas, Suède) avait abouti à la conclusion que seul le Japon avait réalisé des gains de productivité légèrement supérieurs à ceux l'Allemagne. Et elle était parvenue au constat suivant : en se traduisant par une baisse de la tarification des services, ces gains de productivité avaient principalement bénéficié à la clientèle des instituts financiers - et ce, dans une proportion atteinte dans aucun autre des pays étudiés.

Mettre à la disposition des entreprises et des particuliers, à prix accessibles, une gamme la plus complète possible de produits et prestations, autrement dit : assumer pleinement sa fonction d'intermédiation dans l'allocation des capitaux et la transformation des risques - voilà à quoi se résume la mission d'un secteur bancaire efficient dans l'économie. L'étude de la $\mathrm{KfW}$ parvient donc à la conclusion suivante: "Considérés sous cet angle, les instituts allemands non seulement remplissent pleinement leur mission macro-économique au plan national, mais elles sont bien positionnées également en comparaison internationale ».

\section{Le mode de fonctionnement en réseau des caisses d'épargne et des banques coopératives...}

Avec une part de marché cumulée de près de $50 \%$ du total de l'activité bancaire, et un poids supérieur encore dans le segment de la seule banque de détail (plus de $57 \%$ cumulés dans le crédit aux entreprises et près de $64 \%$ dans les opérations de dépôt des particuliers), le réseau des caisses d'épargne Sparkassen-Finanzgruppe et celui des banques coopératives (FinanzVerbund) sont des acteurs majeurs et, plus encore, structurants pour le marché bancaire allemand. Et leur présence (comme leur succès commercial) est le principal signe distinctif du marché allemand en comparaison internationale. Les deux réseaux présentant des caractéristiques similaires, nous nous concentrerons ici sur l'organisation, la culture et le rôle dans l'économie allemande de la Sparkassen-Finanzgruppe.

Les 446 caisses d'épargne locales (état à la fin 2007) constituent la base du groupe. Etablissements autonomes de droit public [ce qui signifie qu'elles exercent une mission d'intérêt général, mais sans que l'Etat soit propriétaire de leur capital ou exerce une tutelle sur elles; IB], elles servent un marché de taille locale ou régionale selon qu'elles ont été instituées par la commune ou le district où elles sont implantées. Elles ne peuvent ouvrir de guichets que dans cette zone d'activité pour servir la clientèle du cru en vertu du principe de la territorialisation de certains services d'intérêt économique général (Regionalprinzip).

Cette configuration en des entités de taille (relativement) faible présente un certain nombre d'avantages. Leur statut implique qu'elles sont entièrement responsables de l'évolution de leur bilan, ce qui entretient non seulement une incitation à l'efficience pour leur management, mais permet aussi une grande réactivité dans la prise de décisions, en particulier dans les opérations de crédit aux entreprises du Mittelstand. La connaissance intime qu'ont les caisses d'épargne des réalités locales (marché, structure des activités...), ainsi que de leur clientèle crée un fort lien de proximité dans la gestion des opérations, ce qui contribue considérablement à réduire l'asymétrie dans l'information si souvent constatée dans les grandes organisations. Cette proximité leur permet en
Des gains de productivité qui profitent au client

Un système bancaire globalement efficient

Des réseaux structurants pour l'activité

Les caisses d'épargne, acteurs de proximité, ...

... ont une forte réactivité 
Le relationship-banking réduit le risque-crédit

Pour compenser leur petite taille,...

... les Sparkassen se sont organisées en réseau

Externalisation des fonctions de back-office

Certaines fonctions sont déléguées à l'échelon supérieur

Conciliation des impératifs de proximité et de compétitivité globale effet d'effectuer des opérations de crédit dont la direction d'un grand groupe n'envisagerait jamais de prendre la responsabilité, le plus souvent tout simplement parce qu'un monitoring aussi fin de la clientèle est impossible dans une structure centralisée, forcément éloignée des réalités du terrain.

Acteurs de terrain, les caisses d'épargne contribuent ainsi au développement de l'activité sans accroître les risques et donc sans générer une hausse du ratio des pertes sur créances. Pour cette raison, chez les membres des réseaux (caisses d'épargne et banques coopératives), le niveau des provisions pour risque-crédit est inférieur à ce qu'il est dans les grandes banques. Avec leur clientèle de particuliers, les caisses d'épargne pratiquent le même mode de relationship banking reposant, comme avec les entreprises, sur une intime connaissance des dossiers et une relation de confiance avec le client. Etablie sur le long terme et donc peu sensible aux variations cycliques, une telle relation avec le client réduit les coûts de transaction pour toutes les parties.

Comme toutes les petites entreprises, les caisses d'épargne tirent donc leur avantage compétitif de leur proximité avec le terrain comme avec la clientèle, qui leur assure une grande réactivité. Néanmoins, comme dans tous les secteurs, les effets de taille sont à prendre en considération dans certaines activités bancaires. Ainsi, dans le back-office, la plupart des fonctions de gestion ou de compensation génèrent des coûts fixes importants ; la dégression des coûts ne peut s'y opérer que grâce à des effets de taille. Les mêmes effets de taille sont requis lorsque l'objectif de répartition des risques exige la constitution d'un portefeuille conséquent.

Or les petits instituts indépendants ne sont pas en mesure de réaliser cette dégression des coûts que rend indispensable la concurrence. Et c'est là justement qu'une organisation en réseau prend tout son sens : elle permet, en les reliant, de cumuler les avantages respectifs d'une petite entité et d'une grande structure, tout en réduisant dans le même temps significativement les handicaps respectifs.

Le pivot en est l'externalisation des fonctions de back-office et leur concentration sous forme d'unités dédiées, entreprises spécialisées communes à tout le réseau. L'exemple le plus patent est celui des ressources informatiques. Au sein de la Sparkassen-Finanzgruppe, les deux centres de calcul qui demeuraient après les restructurations de ces dernières années, et qui figurent parmi les plus importants d'Europe de par leur taille, vont prochainement être fusionnés. Concrètement, cela signifie que chaque caisse d'épargne, aussi petite soit-elle, pourra mettre à profit pour son propre compte l'efficience comme les avantages compétitifs d'un des plus puissants réseaux mondiaux d'ingénierie dans la banque de détail. Le même principe s'applique pour les règlements ou la gestion d'actifs, ainsi que pour le rating conformément aux règles de Bâle II, pour lesquelles la Sparkassen-Finanzgruppe a créé une société ad hoc. Celle-ci gère et développe, pour l'ensemble du réseau, le processus de notation interne commun à toutes les caisses d'épargne et dispose de la plus grande base de données de rating d'entreprises d'Europe ainsi que de la banque de données la plus exhaustive pour le rating des PME. Bien entendu, chaque Sparkasse a un accès direct à ces ressources.

Dans tous les métiers qui, par définition, dépassent le rayon d'action ou les compétences d'une Sparkasse donnée, comme les crédits syndiqués, les opérations sur titres ou le soutien à l'investissement à l'étranger des entreprises allemandes, les établissements ont pour interlocuteurs les banques régionales (Landesbanken) qui, au sein du réseau, assument le rôle de banques universelles ou multimétiers. Pour les opérations centrales que sont la gestion du patrimoine et l'asset management, elles ont plus spécifiquement pour interlocuteur la DekaBank.

Voilà quelques exemples seulement pour illustrer la logique inhérente au fonctionnement en réseau : il s'agit toujours de respecter l'indépendance des acteurs locaux indépendants tout en la conciliant avec une incontournable mise en réseau des fonctions et compétences au niveau de la production comme des 
opérations afin de générer, grâce à l'effet de taille, les économies d'échelle nécessaires pour rester compétitifs. Ce mode d'organisation est le même que celui qu'on observe dans d'autres branches fonctionnant elles aussi en réseau, comme le commerce de détail avec ses points de vente indépendants et sa plateforme centrale d'achat. Le système des franchises présente lui aussi une grande similitude, bien qu'il s'en distingue sur quelques points. Pour le dire autrement : ce mode d'organisation a fait ses preuves partout où il importe de concilier les avantages compétitifs nés d'une part de l'effet de taille, de l'autre, de la proximité avec la demande du marché.

\section{... a des effets bénéfiques sur l'activité en Allemagne}

II n'y a pas que la compétitivité des caisses d'épargne et des banques coopératives qui plaide pour des structures en réseau, surtout dans le métier de la banque de détail. Car elles ont aussi et avant tout des effets bénéfiques sur l'ensemble de l'activité, dont le secret réside justement dans le polycentrisme des acteurs de ces réseaux. Car ce polycentrisme ne se traduit pas seulement par un profond ancrage des établissements dans l'économie réelle à l'échelle locale ; il garantit également la continuité de leur stratégie commerciale, cette continuité étant elle-même la résultante de leur ancrage dans le terroir et des responsabilités qui en découlent pour la prospérité économique de leur zone d'implantation. Etablie sur des relations de confiance, cette approche est donc aussi source de prévisibilité. Pour résumer, la structure en réseau garantit à toutes les catégories de la population et à tous les types de clientèle l'accès aux produits et prestations bancaires; elle intensifie de même la concurrence et contribue de la sorte à la stabilité.

Les structures bancaires polycentriques permettent à tous types de clients l'accès aux produits et prestations bancaires, et ce partout en Allemagne, y compris dans les zones rurales ou les régions en restructuration. Ce n'est donc pas un hasard si, à en croire la Commission européenne, $3 \%$ seulement des Allemands de plus de 18 ans sont considérés comme des exclus du système bancaire (financially excluded) ; c'est l'un des taux les plus bas de l'UE. Et ce n'est pas le fruit du hasard non plus si, en Allemagne, $80 \%$ des bénéficiaires de revenus de transfert publics ont un compte courant dans une caisse d'épargne.

Comme celui des banques coopératives, le réseau des caisses d'épargne couvre la totalité du territoire allemand, grâce à près de 20000 succursales (dont les guichets automatiques). A l'opposé, les grandes banques (hors Postbank) ont fermé leurs guichets dans un district sur dix, et même dans un sur six dans les nouveaux Länder. Et pourtant, même (ou surtout) à l'ère de la banque à distance (via Internet ou le téléphone), les petites et moyennes entreprises ont plus que jamais besoin de l'expertise financière de leur conseiller local...

L'existence de structures polycentriques est l'une des raisons pour lesquelles le marché bancaire allemand se caractérise par une forte concurrence, et sur l'ensemble du territoire. Celle-ci ne se limite en effet pas aux grandes agglomérations ou aux zones de concentration d'activité ; elle joue tout autant dans les zones rurales ou à faible activité. Car même dans les régions que les grandes banques ont délaissées depuis longtemps, il existe au moins une caisse d'épargne et une banque coopérative. Certes, de nombreux opérateurs critiquent régulièrement l'intensité de la concurrence et les faibles marges sur le marché bancaire allemand, allant parfois même jusqu'à considérer cet état de fait comme un « handicap pour le site Allemagne ». Mais il n'y a pas que les clients et consommateurs qui, par définition, s'inscriront en faux contre cette opinion. Car croire que la concurrence constitue un handicap est une thèse qui ne résiste guère non plus à l'analyse macro-économique.

Un autre argument, que nous livre avec une acuité brûlante l'actualité, plaide lui aussi, du point de vue macro-économique, pour des structures polycentriques
Produits et services bancaires sont accessibles à tous...

... et sur l'ensemble du territoire

Le polycentrisme entretient la concurrence...

... et contribue à la stabilité du système bancaire allemand... 
... en forçant l'ancrage des instituts dans l'économie réelle

Garantir la stabilite d'un réseau...

... qui assure une mission d'intérêt général constituées en réseau, tout particulièrement dans la banque de détail. Car ces structures présentent des avantages indéniables pour la stabilité du système financier et bancaire : comme elles sont de petite taille, les caisses d'épargne et les banques coopératives ne peuvent en aucun cas constituer un risque systémique. Comme dans le cas d'un rayon de miel, la simple existence d'une multitude d'alvéoles ou d'établissements suffit à créer la stabilité de l'ensemble.

Autre facteur de stabilité : du fait de leur proximité avec le client, les instituts locaux ou régionaux sont mieux à mêmes que la lointaine centrale d'un grand groupe de gérer les risques au plus près et de décider plus rapidement, et en toute connaissance de cause, d'accorder un crédit ou non. Enfin, et c'est ce qu'ont révélé les travaux dans le cadre de la mise en œuvre des dispositifs de Bâle II : l'implication à large échelle des instituts dans le retail banking se traduit par des portefeuilles de crédits très diversifiés et des encours largement disséminés, donc par une structure favorable du risque crédit.

Enfin, l'ancrage des instituts dans le terroir les contraint à intégrer intimement leurs activités financières dans la structure locale de l'économie réelle. De même, les liens de confiance que crée cette proximité entre l'entreprise et sa banque favorisent une culture axée sur le long terme, ce qui accroît la prévisibilité des affaires pour les deux parties. Cette étroite imbrication barre la route à ces tendances à la volatilité ou à l'émergence de bulles spéculatives qui se nourrissent de cette 'propension à l'anticipation' et de ce 'comportement moutonnier' caractéristiques d'une sphère financière sans lien avec l'économie réelle.

La crise financière actuelle a révélé à quel point c'est la structure même des caisses d'épargne et des banques coopératives qui les a préservées du risque de recourir aux subprimes. De même, elles disposent d'une réserve suffisante en matière de capital et de liquidités pour continuer à exercer et même à développer leur métier de bailleur de fonds. Ainsi, au cours du premier semestre 2008, les seules Sparkassen ont octroyé près de 24,5 milliards $€$ de nouveaux crédits à leurs clientèle du Mittelstand. Ce volume est en hausse de 11,5\% par rapport au premier semestre 2007. On ne peut donc parler d'un quelconque risque de credit crunch auquel seraient exposées les PE allemandes.

\section{Les défis que doit relever le réseau des Sparkassen}

La compétitivité de la Sparkassen-Finangruppe, mais aussi de son homologue des banques coopératives, le FinanzVerbund, et, plus important encore, l'impact positif de ces réseaux sur l'ensemble de l'activité en Allemagne révèle qu'une telle organisation en réseau, parce qu'elle est chargée de sens, constitue incontestablement non seulement un modèle économique performant pour ses membres, mais présente aussi des avantages indéniables pour l'économie allemande. Ces structures et ce système doivent donc être pérennisés. Or pour garantir les performances de ces réseaux dans le futur, il faut en préserver la source de stabilité : la structure d'ensemble du réseau, et veiller à ne pas en exclure hâtivement certains éléments.

La stabilité d'un tel réseau coopératif repose en effet sur le principe du "one man, one vote ". Dans le cas des Sparkassen, leur statut " d'établissement de droit public " (Anstalt öffentlichen Rechts) a fait ses preuves au fil des décennies. Ce statut permet aux caisses d'épargne allemandes de se positionner dans la concurrence, comme n'importe quelle autre entreprise sur le marché, et $\mathrm{ce}$, sans que ce statut s'accompagne de privilèges ou de garanties d'Etat. Etablissement de droit public n'est donc, en l'occurrence, nullement synonyme de propriété d'Etat, de tutelle gouvernementale, d'inefficience ou d'entrave à la concurrence. Ce statut est bien au contraire l'instrument adéquat permettant de garantir à tous l'accès aux services financiers, d'assurer un haut niveau de concurrence favorable au client et de garantir la stabilité globale de l'économie. 


\begin{abstract}
A qui appartiennent les Sparkassen?
A personne, c'est-à-dire à tout le monde, en réalité. L'origine de leur statut particulier remonte à 1931 : durant la crise bancaire de l'époque, elles avaient été sorties du périmètre du patrimoine communal, et dotées d'un statut autonome (au plan juridique comme en matière de gestion) pour interdire aux trésoriers communaux de puiser dans leurs actifs. Comme elles exercent une mission d'intérêt économique général, elles ont été dotées d'un statut de « collectivité publique ", proche de celui d'une fondation dans le secteur privé ou marchand. Ce statut public, émanation concrète de la mission impartie à un tel établissement, se traduit par le fait qu'une Sparkasse, en l'occurrence, ne peut appartenir ni à l'Etat (ici l'exécutif communal) ni à des intérêts privés (investisseur). Ce statut étant fondé sur le principe de subsidiarité, une Sparkasse 'appartient' donc en quelque sorte à la collectivité. Ou, comme le formule sa fédération : "aux citoyens ». (IB)
\end{abstract}

Et pourtant, ce statut de droit public se trouve régulièrement sous le feu des critiques. A commencer par celles des banques privées allemandes. Et même le Conseil des Sages (Sachverständigenrat), qui est l'autorité indépendante en charge de l'évaluation de l'évolution macro-économique, préconise dans un récent rapport d'expertise consacré à la finance allemande (Sachverständigenrat, 2008), la transformation des caisses d'épargne an sociétés par actions et l'ouverture de leur capital au privé.

Cette recommandation ne résiste cependant pas à l'analyse, d'autant que dans le même rapport, le Conseil des Sages souligne expressément l'avantage que présente une structure d'acteurs polycentriques constitués en réseau pour le financement du Mittelstand, pour la concurrence et pour la stabilité du système financier en Allemagne. Ce qui revient à plaider pour le maintien de cette structure. Or la mise en œuvre de ses préconisations concernant le statut des Sparkassen irait à l'encontre de cette recommandation. C'est la raison pour laquelle l'avis du Conseil des Sages a reçu un accueil pour le moins mitigé dans les milieux politiques, certains allant jusqu'à s'y opposer fermement. Ainsi, le ministre fédéral des Finances, Peer Steinbrück, a récemment encore rappelé sans équivoque l'opposition du gouvernement fédéral à toute modification du statut d'établissement de droit public qu'ont les caisses d'épargne allemandes (Bundesministerium der Finanzen, 2008).

Bien que bénéficiant d'un tel soutien politique, le groupe Sparkassen-Finanzgruppe doit bien évidemment relever d'importants défis économiques et commerciaux pour rester compétitif sur un marché où la concurrence ne cesse de s'intensifier. Le cœur de sa stratégie est dès lors de poursuivre le regroupement des fonctions de back-office, c'est-à-dire de réaliser des gains d'efficience grâce à une politique de consolidation menée sous l'approche d'une "fusion des process ».

Mais le chantier le plus important et le plus urgent actuellement au sein du groupe est celui des banques régionales, les Landesbanken. L'évolution de ces derniers mois a en effet clairement confirmé que le volume des opérations résultant de la coopération avec les caisses d'épargne est insuffisant en comparaison des capacités effectives dont disposent aujourd'hui les Landesbanken. Or dans le même temps, le marché n'offre à ces dernières que très peu de possibilités pour se diversifier dans d'autres segments du métier de banque d'affaires. Certaines Landesbanken en avaient conclu qu'il leur fallait s'investir plus dans les opérations d'une finance internationale sans lien avec l'économie réelle. L'actualité de ces derniers mois a mis sous le feu des projecteurs les risques inhérents à une telle stratégie et placé les Landesbanken quelque peu sous contrainte.

Ce constat a incité les parties prenantes des Landesbanken que sont les caisses d'épargne à adopter une position ferme. Elles attendent ainsi des banques régionales que celles-ci les soutiennent mieux que par le passé dans l'ensemble de leur palette de prestations-clients et qu'elles visent désormais un objectif de rentabilité plus conforme à leur développement durable. Cet objectif prôné par les Sparkassen signifie que les banques régionales doivent limiter le volume de leurs
Un statut public souvent critiqué...

... mais ardemment défendu par le gouvernement fédéral

Réaliser des gains d'efficience

Moderniser structure et missions des Landesbanken

Les Sparkassen exigent qu'elles suivent une stratégie moins spéculative... 
... et qu'elles soient amenées à fusionner opérations sur les marchés des valeurs à haut risque - elles sont en effet contraires à l'approche conservatrice des affaires et de la gestion du risque qui fait l'identité des caisses d'épargne. En tant que co-actionnaires et co-responsables (devant les tribunaux) des Landesbanken, les caisses d'épargne attendent donc de celles-ci qu'elles établissent à l'avenir leur stratégie de rentabilité sur d'autres bases que celle des valeurs mobilières à forte volatilité.

Cela passe par une réforme structurelle des Landesbanken. Car si leur rôle-clef en tant que partenaires des caisses d'épargne au sein de leur réseau est indéniable, rien n'exige en revanche que soient maintenus en Allemagne sept groupes autonomes de banques publiques régionales. Leur concentration permettrait non seulement de rendre leur modèle économique plus performant, mais il réduirait aussi pour elles l'incitation à s'engager dans des opérations à forte volatilité, ce qui, au final, consoliderait durablement l'ensemble du réseau Sparkassen-Finanzgruppe. Mais en aucune façon, la solution ne doit consister à ce que les caisses d'épargne injectent un surcroît de capital dans les Landesbanken, ni encore moins à intégrer certaines Sparkassen dans le périmètre des banques régionales. Car une telle 'intégration verticale' finirait irrémédiablement par réduire à néant le facteur structurel qui fait la force de la SparkassenFinanzgruppe, à savoir son polycentrisme.

LE MARCHE BANCAIRE ALLEMAND AUSSI est aux prises avec de profondes mutations structurelles, et ce depuis de longues années. Les récentes fusionsacquisitions annoncées dans le secteur des grandes banques privées révèlent que ce processus est loin d'être achevé. Et malgré le profond besoin de modernisation du dispositif des banques publiques régionales, il n'en reste pas moins que, globalement, le système bancaire allemand présente des atouts indéniables. Ses atouts résident dans une concurrence intense, une haute efficience et une grande stabilité. Ils résultent essentiellement du polycentrisme des acteurs réunis au sein de la Sparkassen-Finanzgruppe et du FinanzVerbund. Cette structuration en réseau constitue, surtout en ce qui concerne la banque de détail, un modèle économique compétitif pour chacun des instituts membres et, par delà, un mode de fonctionnement non seulement adapté, mais aussi bénéfique à l'ensemble des activités. De ce fait, la logique de réseau se révèle également une base saine pour assurer, à l'avenir aussi, la compétitivité d'un système dans un environnement de plus en plus concurrentiel.

Traduction : I. Bourgeois

\section{Indications bibliographiques}

BANQUE CENTRALE EUROPEENNE, EU Banking Structures, octobre 2007

BANQUE CENTRALE EUROPEENNE, MFI interest rate statistic, septembre 2008

BundeSMINISTERIUM DER FINANZEN, «Keine Schulden. Alle Chancen », discours du ministre fédéral des Finances lors de la première lecture au Bundestag du projet de loi de finances 2009, 16-09-2008

STEINBRÜCK P., «Ein Erdbeben mit unvorstellbaren Wertberichtigungen », déclaration gouvernementale sur la situation des marchés financiers faite au Bundestag le 25-092008, Das Parlament, 29-9/06-10-2008

Deutsche Bundesbank, Bankenstatistik, Statistisches Beiheft zum Monatsbericht, août 2008

Deutsche Bundesbank, « Die Ertragslage der deutschen Kreditinstitute im Jahr 2007 », in Monatsbericht, septembre 2008

DEUTSCHE BUNDESBANK, « Die Ertragslage der deutschen Kreditinstitute im Jahr 2006 », in Monatsbericht, septembre 2007

Initiative Finanzstandort Deutschland, rapport $n^{\circ} 4,2008$

KFW-RESEARCH, Das deutsche Kreditgewerbe im internationalen Vergleich, Francfort, 2005

OLIVER Wyman, Analysen Europa - Ergebnisse des Preisbenchmarking, 2008

MERCER OLIVER WYMAN, European Mortgage Markets, février 2007

SACHVERSTÄNDIGENRAT ZUR BEGUTACHTUNG DER GESAMTWIRTSCHAFTLICHEN ENTWICKLUNG, Das deutsche Finanzsystem, Effizienz steigern - Stabilität erhöhen, Rapport d'expertise réalisé pour le compte du gouvernement fédéral, juin 2008. 